\title{
ON THE ACCURACY AND CONVERGENCE OF THE MINIMAX FILTERING ALGORITHM FOR CHAOTIC SIGNALS
}

\author{
A. S. Sheludko, South Ural State University, Chelyabinsk, Russian Federation, \\ sheludkoas@susu.ru.
}

The article is focused on the filtering problem for chaotic signals. The original discrete signal is generated by a one-dimensional chaotic system, and the measured signal is corrupted by additive errors. The goal is to estimate the unknown system states from measurements. The minimax filtering algorithm is developed in the context of guaranteed state estimation that is based on a set-membership description of uncertainty. It assumes that the unknown variables (system states and measurement errors) are bounded by intervals (sets of possible values). The proposed algorithm is a recursive procedure based on interval analysis. It computes interval estimates that are guaranteed to contain the true states of the system (true values of the original signal). The computation of the interval estimate consist of three steps (prediction, measurement, and correction) that are similar to the computation of the information set for linear dynamical systems. The point estimates are obtained by an algorithm that is similar to the Kalman filter. This paper studies the accuracy and convergence properties of the minimax filter. The aims of this study are the following: to confirm the effectiveness of the proposed algorithm for computation of the point estimates, to compare the results of the minimax filter and the unscented Kalman filter, and to derive the sufficient conditions for obtaining the exact value of the state. The computational scheme of the minimax filter and numerical simulations are given for the logistic map.

Keywords: chaotic signal; filtering problem; guaranteed estimation; interval estimate.

\section{Introduction}

The foundation of modern filtering theory is related to the state estimation problem for linear systems. Based on the probabilistic description of the unknown variables, the Kalman filter (KF) has become the most important and widely used technique in signal processing [1]. Various modifications of the $\mathrm{KF}$ have been proposed for nonlinear systems [2,3]. State estimation problem has also become a starting point for the development of guaranteed approach, which is based on a set-membership description of uncertainty $[4,5]$. As a result, an increasing interest has been devoted to guaranteed state estimation using different kinds of set representation, such as ellipsoids [6], parallelotopes [7], boxes [8], and zonotopes [9].

This article is focused on the filtering problem for chaotic signals [10-12]. The considered problem is an important one in chaos-based communications and signal processing [13-15]. It is also a useful tool in the development of the parameter estimation techniques (for example, see the approach proposed in [16]).

Consider a one-dimensional chaotic system described by

$$
x_{k}=f\left(x_{k-1}, \lambda\right),
$$

where $f$ is the chaotic map (see definition in [17]), $x_{k}$ is the system state, $\lambda$ is the map parameter, and $k$ is the number of time step. System (1) generates the original discrete 
signal. The measured signal is given by

$$
y_{k}=x_{k}+v_{k}, k=1,2, \ldots, N
$$

where $y_{k}$ is the measurement, $v_{k}$ is the additive error, and $N$ is the number of measurements.

The filtering problem is to estimate the states of the system (1) from measurements (2). The most common approach is to use modifications of the KF for nonlinear systems, such as the extended KF [10], the unscented KF [11], and the cubature KF [12]. Recently in [18], the minimax filter (MF) was developed in the context of guaranteed estimation. This article studies the accuracy and convergence properties of the MF.

\section{The Algorithm of the Minimax Filter}

The MF assumes that the initial state $x_{0}$ and measurement errors $v_{k}, k=1,2, \ldots, N$ are bounded by intervals:

$$
x_{0} \in X_{0}=\left[\underline{x}_{0}, \bar{x}_{0}\right], v_{k} \in V_{k}=\left[\underline{v}_{k}, \bar{v}_{k}\right], k=1,2, \ldots, N .
$$

The result of the MF at time step $k$ is the interval estimate $X_{k}$ of the state $x_{k}$ and the point estimate $\hat{x}_{k} \in X_{k}$. If the restrictions (3) are correct, it is guaranteed that the found interval $X_{k}$ contains the true value $x_{k}^{*}$ of the state $x_{k}$ :

$$
x_{k}^{*} \in X_{k}=\left[\underline{x}_{k}, \bar{x}_{k}\right] .
$$

Computation of the interval estimates. The interval estimates $X_{k}, k=1,2, \ldots, N$ are defined recursively. The computation consist of three steps: prediction, measurement, and correction. This algorithm is similar to the computation of the information set for linear dynamical systems [5].

Step 1 (prediction). The predicted state set $X_{k / k-1}$ is defined by the interval estimate $X_{k-1}$ found at the previous time step:

$$
X_{k / k-1}=\left\{u \mid u=f(x, \lambda), x \in X_{k-1}\right\} .
$$

Step 2 (measurement). The consistent state set $Y_{k}$ is defined by the measurement $y_{k}$ and the interval estimate $V_{k}$ of the measurement error $v_{k}$ :

$$
\begin{aligned}
Y_{k} & =\left\{x \mid x=y_{k}-v, v \in V_{k}\right\}= \\
& =\left\{x \mid y_{k}-\bar{v}_{k} \leq x \leq y_{k}-\underline{v}_{k}\right\}= \\
& =\left[y_{k}-\bar{v}_{k}, y_{k}-\underline{v}_{k}\right] .
\end{aligned}
$$

Step 3 (correction). The interval estimate $X_{k}$ of the state $x_{k}$ is the intersection of the predicted state set $X_{k / k-1}(4)$ and the consistent state set $Y_{k}(5)$ :

$$
X_{k}=X_{k / k-1} \cap Y_{k}
$$

Computation of the point estimates. An important problem in guaranteed estimation is how to derive point estimates from the interval estimates [4]. Usually a point estimate 
$\hat{\mathbf{u}}$ of the unknown vector $\mathbf{u} \in U \subset \mathbb{R}^{n}$ is obtained by the minimization of the worst-case error [5]:

$$
\min _{\hat{\mathbf{u}} \in U} \max _{\mathbf{u} \in U}\|\mathbf{u}-\hat{\mathbf{u}}\|,
$$

where $U$ is the set of possible values of the vector $\mathbf{u}$. In the one-dimensional case, the solution of the minimax problem (7) is the middle point of the interval. However, numerical simulations show that the true value $x_{k}^{*}$ of the state $x_{k}$ is often close to the bounds of the interval estimate $X_{k}$. Thus, the choice of the middle point

$$
\hat{x}_{k}=\frac{\underline{x}_{k}+\bar{x}_{k}}{2}
$$

as a point estimate may not be effective. Recently in [18], an algorithm that is similar to the KF was proposed to obtain the point estimates.

Suppose that $\hat{x}_{0} \in X_{0}$ is the prior estimate for $k=0$. The point estimates $\hat{x}_{k}, k=1,2, \ldots, N$ are defined recursively by the following steps.

Step 1. Computation of the predicted state estimate:

$$
\hat{x}_{k / k-1}=f\left(\hat{x}_{k / k-1}, \lambda\right)
$$

Step 2. Computation of the gain coefficient:

$$
l_{k}=\frac{\Delta X_{k / k-1}}{\Delta X_{k / k-1}+\Delta V_{k}},
$$

where $\Delta$ denotes the length of the interval.

Step 3. Correction of the state estimate:

$$
\tilde{x}_{k}=\hat{x}_{k / k-1}+l_{k}\left(y_{k}-\hat{x}_{k / k-1}\right)
$$

Step 4. Verification of the state estimate. If $\tilde{x}_{k} \notin X_{k}$, then the point estimate $\hat{x}_{k}$ is defined as the closest bound of the interval $X_{k}$ :

$$
\hat{x}_{k}= \begin{cases}\tilde{x}_{k}, & \tilde{x}_{k} \in X_{k}, \\ \underline{x}_{k}, & \tilde{x}_{k}<\underline{x}_{k}, \\ \bar{x}_{k}, & \tilde{x}_{k}>\bar{x}_{k}\end{cases}
$$

\section{Numerical Simulations}

The chaotic signal is generated by the logistic map

$$
x_{k}=\lambda x_{k-1}\left(1-x_{k-1}\right)
$$

with the initial state $x_{0}=0.2$ and parameter $\lambda=3.7$ (Fig. 1). The measured signal is shown in Fig. 2. The number of measurements is $N=20$. The measurement errors are pseudo-random numbers with normal distribution, zero mean and standard deviation $\sigma_{v}=0.05$ (Fig. 3). The values of $v_{k}$ are generated by the function randn of Matlab. 


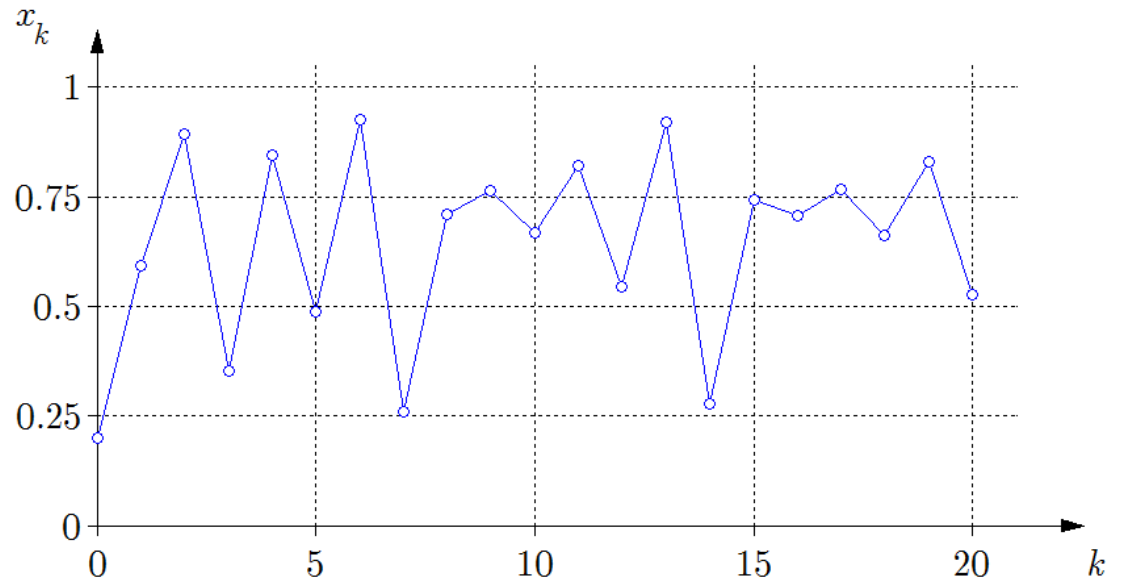

Fig. 1. Chaotic signal generated by the logistic map

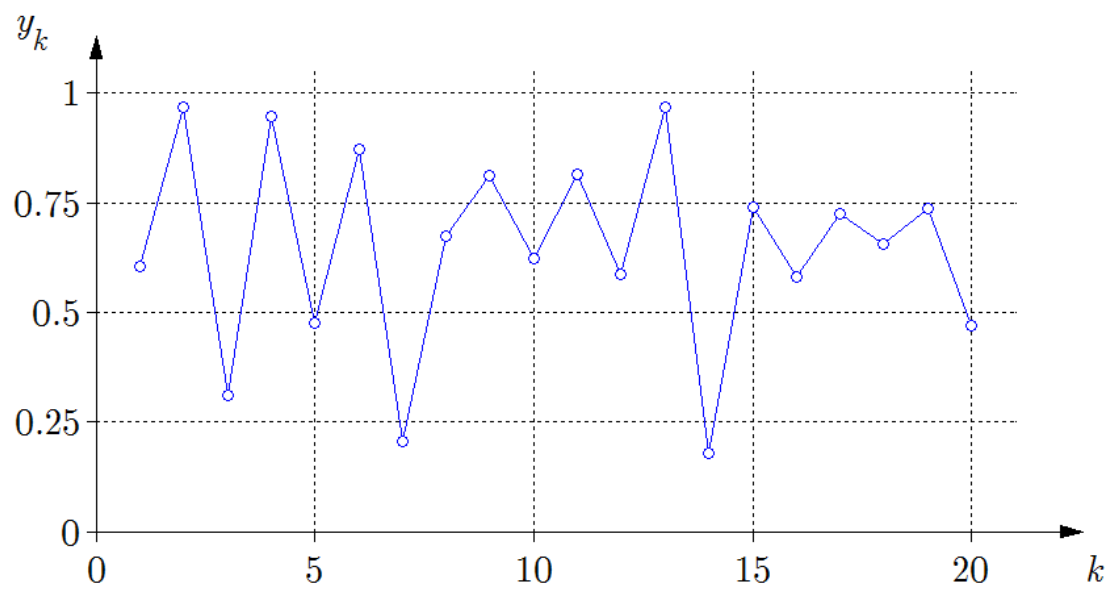

Fig. 2. Measured signal corrupted by additive errors

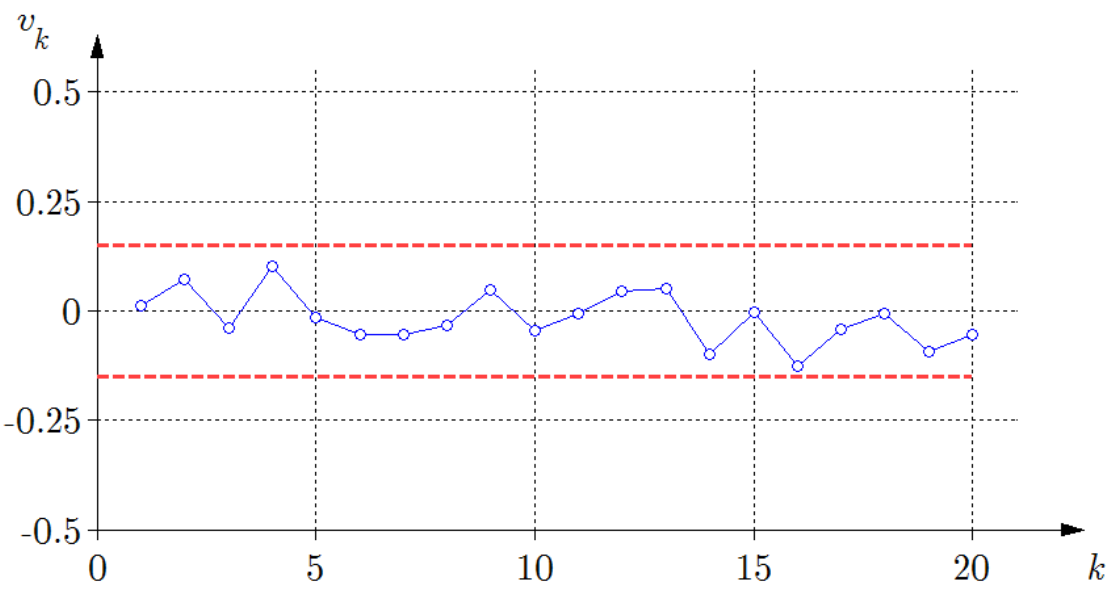

Fig. 3. Measurement errors (dashed lines denote the bounds of the interval estimate) 
In this example, the signal-to-noise ratio is

$$
\mathrm{SNR}=20 \lg \frac{\sigma_{x}}{\sigma_{v}}=12 \mathrm{~dB},
$$

where $\sigma_{x}=0.1976$ is the sample standard deviation of the chaotic signal. The maximum absolute value of the measurement error is $\max \left|v_{k}\right|=0.1259$.

The prior estimates are taken as follows:

$$
\hat{x}_{0}=0.25, X_{0}=[0,0.5], V_{1}=\cdots=V_{N}=\left[-3 \sigma_{v}, 3 \sigma_{v}\right]=[-0.15,0.15] .
$$

The computation of the predicted state set $X_{k / k-1}=\left[\underline{x}_{k / k-1}, \bar{x}_{k / k-1}\right]$ (4) for the logistic map (13) can be represented by the following "if-then" rules (Fig. 4):

1. If $\bar{x}_{k-1} \leq \frac{1}{2}$ then $\underline{x}_{k / k-1}=\lambda \underline{x}_{k-1}\left(1-\underline{x}_{k-1}\right), \bar{x}_{k / k-1}=\lambda \bar{x}_{k-1}\left(1-\bar{x}_{k-1}\right)$.

2. If $\underline{x}_{k-1} \geq \frac{1}{2}$ then $\underline{x}_{k / k-1}=\lambda \bar{x}_{k-1}\left(1-\bar{x}_{k-1}\right), \bar{x}_{k / k-1}=\lambda \underline{x}_{k-1}\left(1-\underline{x}_{k-1}\right)$.

3. If $\underline{x}_{k-1}<\frac{1}{2}$ and $\bar{x}_{k-1}>\frac{1}{2}$ then:

3.1. If $\underline{x}_{k-1}<1-\bar{x}_{k-1}$ then $\underline{x}_{k / k-1}=\lambda \underline{x}_{k-1}\left(1-\underline{x}_{k-1}\right), \bar{x}_{k / k-1}=\frac{\lambda}{4}$.

3.2. If $\underline{x}_{k-1} \geq 1-\bar{x}_{k-1}$ then $\underline{x}_{k / k-1}=\lambda \bar{x}_{k-1}\left(1-\bar{x}_{k-1}\right), \bar{x}_{k / k-1}=\frac{\lambda}{4}$.

The complexity of the MF implementation depends on the certain equation of the chaotic map. In general, the development of a computational scheme is based on interval analysis [8].

Consider the computation of the interval estimates $X_{1}$ and $X_{2}$ by the algorithm (4)-(6). According to the above scheme, the predicted state set $X_{1 / 0}$ is defined as

$$
X_{1 / 0}=\left[\lambda \underline{x}_{0}\left(1-\underline{x}_{0}\right), \lambda \bar{x}_{0}\left(1-\bar{x}_{0}\right)\right]=[0,0.9250] .
$$

The measured value $y_{1}=0.6032$, and the interval estimate of the error $v_{1}$ is $V_{1}=[-0.15,0.15]$. Thus, according to (5) the consistent state set is

$$
Y_{1}=\left[y_{1}-\bar{v}_{1}, y_{1}-\underline{v}_{1}\right]=[0.4532,0.7532] \text {. }
$$

Therefore, at time step $k=1$ the interval estimate $X_{1}$ of the state $x_{1}$ is

$$
X_{1}=X_{1 / 0} \cap Y_{1}=[0,0.9250] \cap[0.4532,0.7532]=[0.4532,0.7532] .
$$

Since $\underline{x}_{1}<\frac{1}{2}, \bar{x}_{1}>\frac{1}{2}$, and $\underline{x}_{1}>1-\bar{x}_{1}$ the predicted state set $X_{2 / 1}$ is defined as

$$
X_{2 / 1}=\left[\lambda \bar{x}_{1}\left(1-\bar{x}_{1}\right), \lambda / 4\right]=[0.6877,0.9250] .
$$

The measured value $y_{2}=0.9663$, and the interval estimate of the error $v_{2}$ is $V_{2}=[-0.15,0.15]$. Thus, according to (5) the consistent state set is

$$
Y_{2}=\left[y_{2}-\bar{v}_{2}, y_{2}-\underline{v}_{2}\right]=[0.8163,1.1163] \text {. }
$$

Therefore, at time step $k=2$ the interval estimate $X_{2}$ of the state $x_{2}$ is

$$
X_{2}=X_{2 / 1} \cap Y_{2}=[0.6877,0.9250] \cap[0.8163,1.1163]=[0.8163,0.9250] .
$$



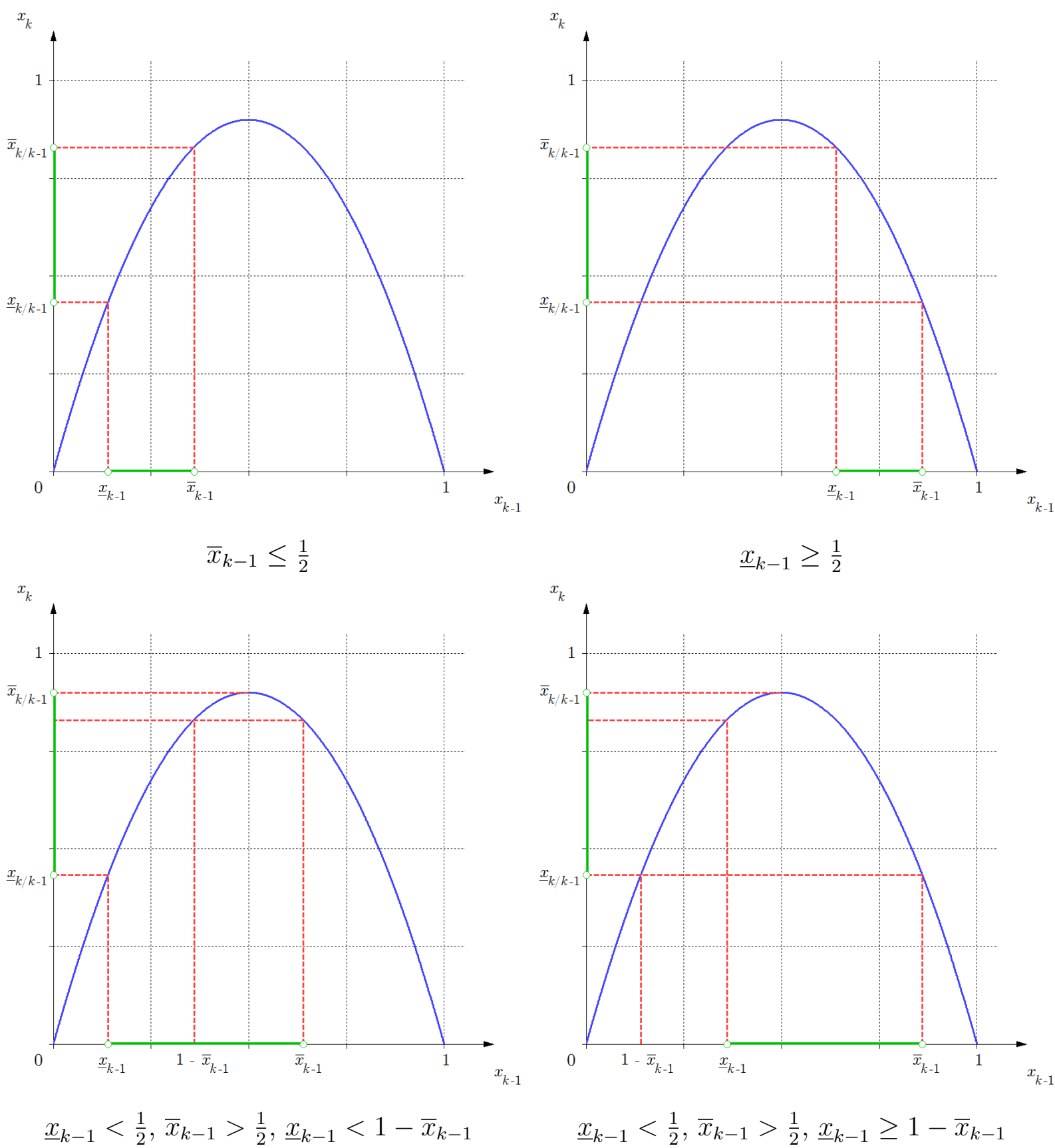

Fig. 4. Computation of the predicted state set for the logistic map

The results of the MF are presented in Fig. 5. As already noticed, the true value $x_{k}^{*}$ of the state $x_{k}$ is often close to the bounds of the interval estimate $X_{k}$. Fig. 6 shows the comparison of the estimation error $\delta_{k}$ and the maximum possible error $\mu_{k}$, which are defined as follows:

$$
\begin{gathered}
\delta_{k}=\left|x_{k}^{*}-\hat{x}_{k}\right|, \\
\mu_{k}=\max _{u \in X_{k}}\left|x_{k}^{*}-u\right| .
\end{gathered}
$$




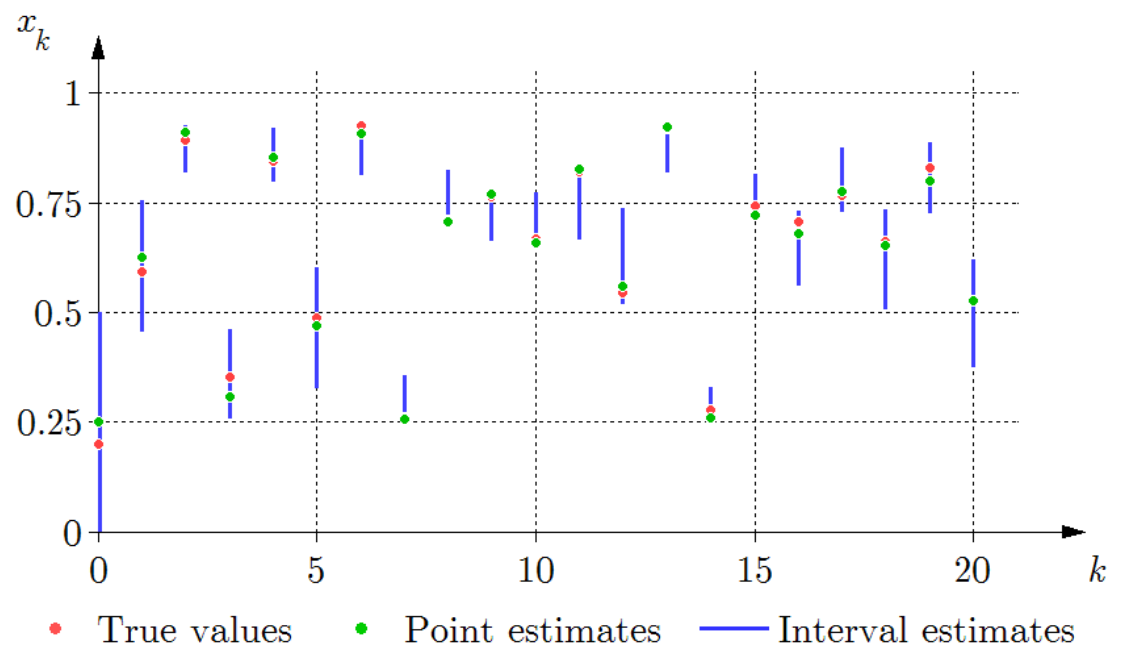

Fig. 5. State estimates computed by the MF

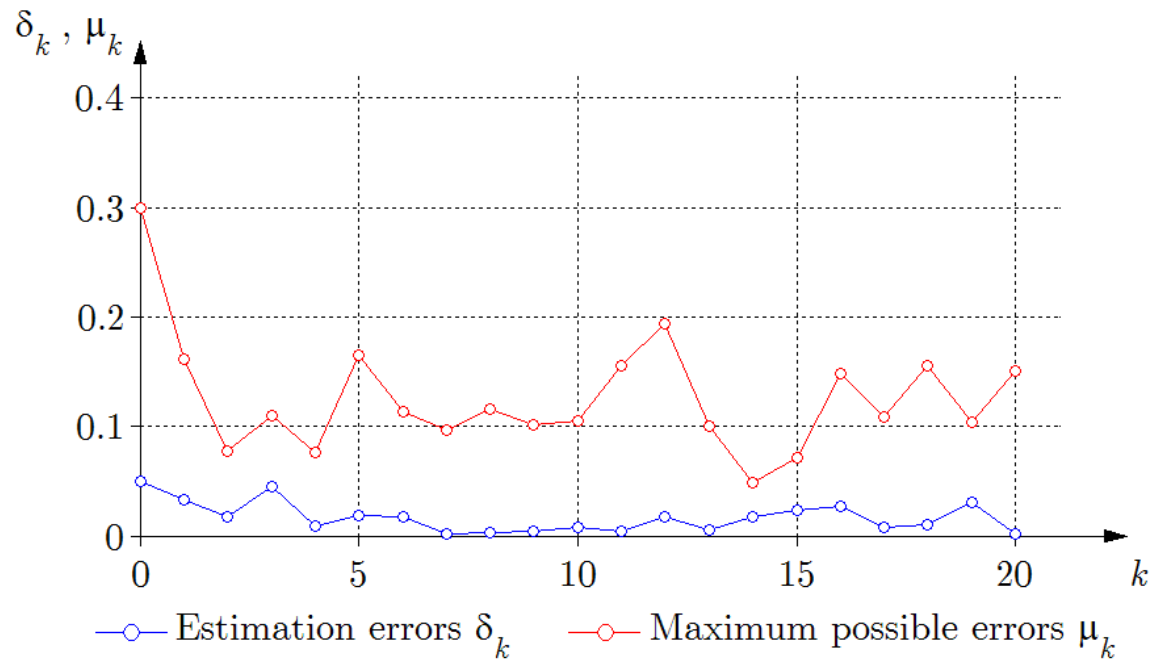

Fig. 6. Estimation errors of the MF

In order to confirm the effectiveness of the proposed algorithm (9)-(12) for computation of the point estimates, the results are averaged over $n=10^{4}$ runs with different initial condition $x_{0} \in(0,0.5)$ and parameter $\lambda \in[3.6,4]$. The values of $x_{0}$ and $\lambda$ are generated by the function rand of Matlab. In each simulation, the prior estimates are as in the above example. Fig. 7 shows the comparison of the relative error

$$
\epsilon_{k}=\frac{\delta_{k}}{\mu_{k}} \times 100 \%
$$

for the proposed algorithm and in the case when the point estimate is defined as the middle point of the interval (8). 


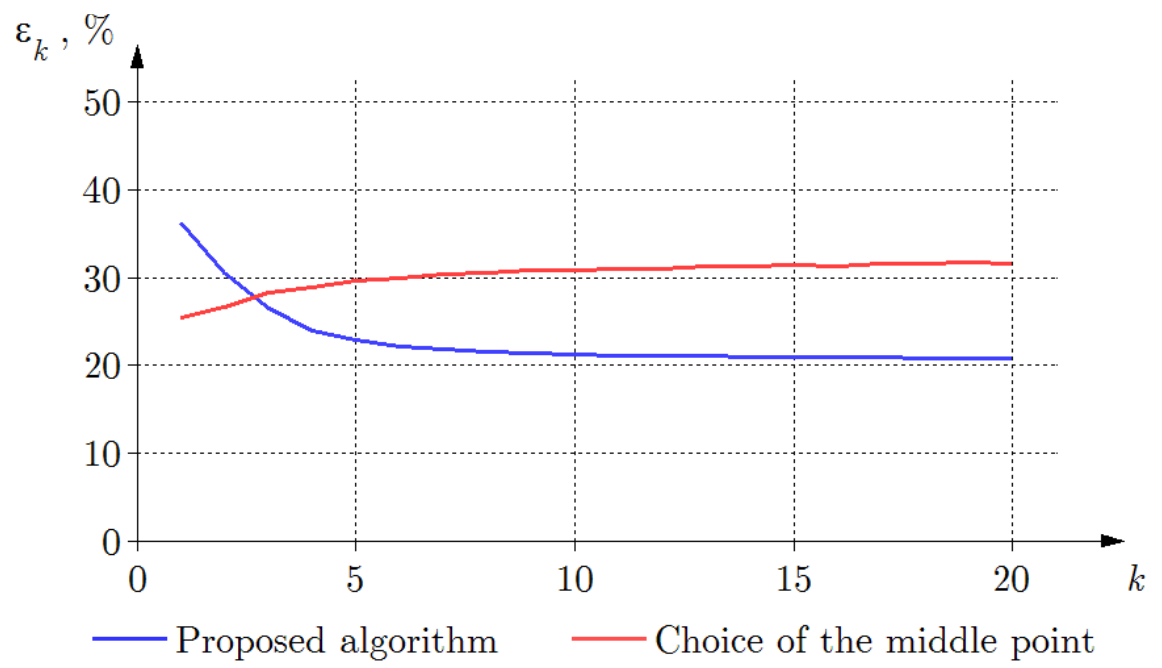

Fig. 7. Comparison of the relative errors for the versions of the MF (the results are averaged over $n=10^{4}$ runs)

\section{Application of the Unscented Kalman Filter}

One of the most common KF modifications for chaotic systems is the unscented Kalman filter (UKF) $[3,11]$. The KF approach is based on the following assumptions:

1. The initial state $x_{0}$ is a random variable, normally distributed with mean $\hat{x}_{0}$ and variance $p_{0}$ :

$$
E\left[x_{0}\right]=\hat{x}_{0}, E\left[\left(x_{0}-\hat{x}_{0}\right)^{2}\right]=p_{0} .
$$

2. The measurement errors $v_{k}, k=1,2, \ldots, N$ are white Gaussian noise with zero mean and standard deviation $\sigma_{v}$ :

$$
E\left[v_{k}\right]=0, E\left[v_{k}^{2}\right]=\sigma_{v}^{2}
$$

The result of the UKF at time step $k$ is the state estimate $\hat{x}_{k}$ and the error variance $p_{k}$ that provides the confidence interval:

$$
S_{k}=\left[\hat{x}_{k}-3 \sqrt{p_{k}}, \hat{x}_{k}+3 \sqrt{p_{k}}\right] .
$$

The UKF equations for model (1), (2) are given as follows:

$$
\begin{gathered}
\hat{x}_{k / k-1}=\frac{1}{2}\left(f\left(\hat{x}_{k-1}+\sqrt{p_{k-1}}, \lambda\right)+f\left(\hat{x}_{k-1}-\sqrt{p_{k-1}}, \lambda\right)\right), \\
p_{k / k-1}=\frac{1}{4}\left(f\left(\hat{x}_{k-1}+\sqrt{p_{k-1}}, \lambda\right)-f\left(\hat{x}_{k-1}-\sqrt{p_{k-1}}, \lambda\right)\right)^{2}, \\
l_{k}=\frac{p_{k / k-1}}{p_{k / k-1}+\sigma_{v}^{2}} \\
\hat{x}_{k}=\hat{x}_{k / k-1}+l_{k}\left(y_{k}-\hat{x}_{k / k-1}\right), \\
p_{k}=\left(1-l_{k}\right) p_{k / k-1},
\end{gathered}
$$


where $\hat{x}_{k / k-1}$ is the predicted state estimate, $p_{k / k-1}$ is the predicted error variance, and $l_{k}$ is the Kalman gain. Since for the logistic map (13)

$$
\begin{aligned}
& f\left(\hat{x}_{k-1}+\sqrt{p_{k-1}}, \lambda\right)=\lambda\left(\hat{x}_{k-1}\left(1-\hat{x}_{k-1}\right)+\left(1-2 \hat{x}_{k-1}\right) \sqrt{p_{k-1}}-p_{k-1}\right), \\
& f\left(\hat{x}_{k-1}-\sqrt{p_{k-1}}, \lambda\right)=\lambda\left(\hat{x}_{k-1}\left(1-\hat{x}_{k-1}\right)-\left(1-2 \hat{x}_{k-1}\right) \sqrt{p_{k-1}}-p_{k-1}\right),
\end{aligned}
$$

equations (15), (16) can be expressed as follows:

$$
\begin{gathered}
\hat{x}_{k / k-1}=\lambda \hat{x}_{k-1}\left(1-\hat{x}_{k-1}\right)-\lambda p_{k-1}, \\
p_{k / k-1}=\lambda^{2}\left(1-2 \hat{x}_{k-1}\right)^{2} p_{k-1} .
\end{gathered}
$$

Consider the example from section 2. The initial state estimate is $\hat{x}_{0}=0.25$ and variance $p_{0}=\left(\frac{0.25}{3}\right)^{2}=0.0069$. With this values, the confidence interval $S_{0}(14)$ is equal to the prior interval estimate $X_{0}: S_{0}=X_{0}=[0,0.5]$. Fig. 8 shows the state estimates computed by the UKF.

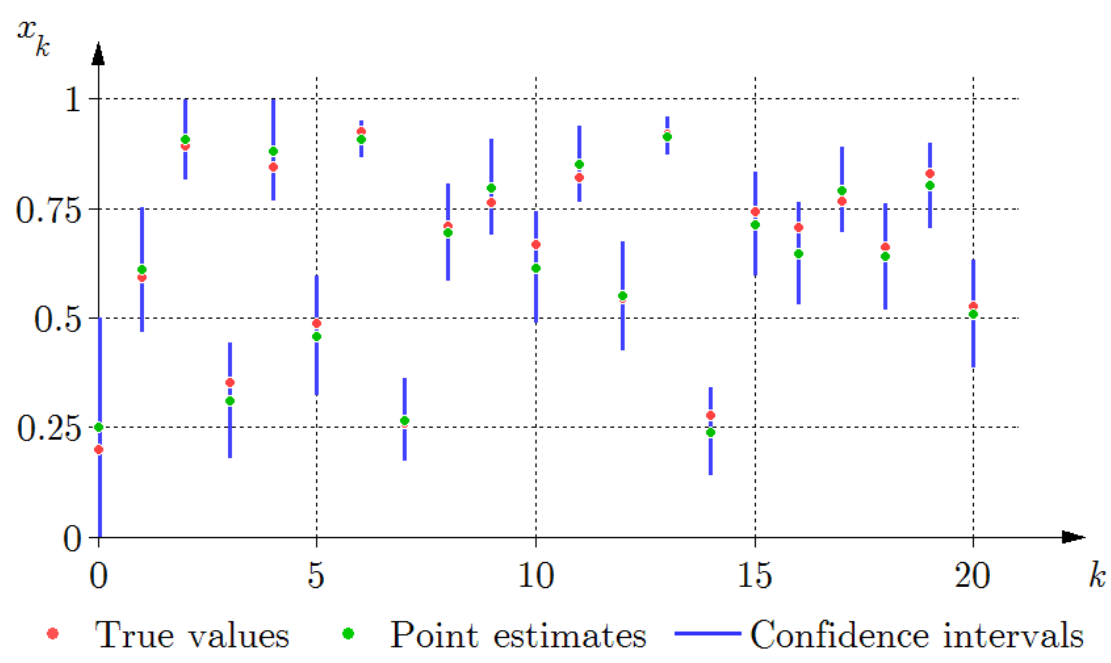

Fig. 8. State estimates computed by the UKF

In order to compare the accuracy of the MF and UKF, the results are averaged over $n=10^{4}$ runs with different initial condition $x_{0} \in(0,0.5)$ and parameter $\lambda \in[3.6,4]$. The values of $x_{0}$ and $\lambda$ are generated by the function rand of Matlab. In each simulation, the initial data are as in the above example. Fig. 9 shows the comparison of estimation errors.

\section{Convergence Analysis of the Algorithm}

This section presents the sufficient conditions under which the MF computes the true state of the system. For simplicity, it is assumed that the interval estimates of measurement errors are fixed:

$$
V_{1}=\ldots=V_{N}=[\underline{v}, \bar{v}] .
$$




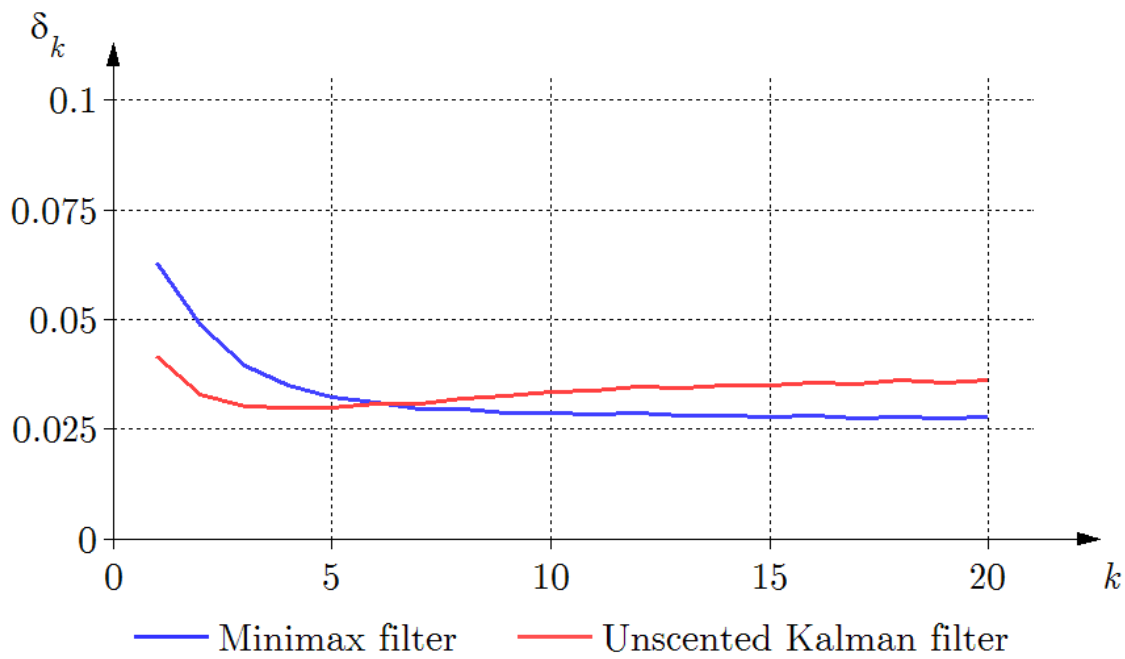

Fig. 9. Comparison of the estimation errors (the results are averaged over $n=10^{4}$ runs)

Theorem 1. Suppose that $f$ is monotone increasing in the predicted state set $X_{k / k-1}$, and the measurement errors at time steps $k$ and $k+1$ are equal to the upper and lower bounds of the interval of possible values, respectively:

$$
v_{k}=\bar{v}, v_{k+1}=\underline{v} .
$$

Then the interval estimate $X_{k+1}$ consists of a single point, which is the true value $x_{k+1}^{*}$ of the state $x_{k+1}$ :

$$
X_{k+1}=\left\{x_{k+1}^{*}\right\}
$$

Proof.

Since $v_{k}=\bar{v}, v_{k+1}=\underline{v}$, the corresponding measured values are

$$
y_{k}=x_{k}+v_{k}=x_{k}^{*}+\bar{v}, y_{k+1}=x_{k+1}+v_{k+1}=x_{k+1}^{*}+\underline{v} .
$$

Thus, according to (5), the consistent state set has the following structure at time steps $k$ and $k+1$ :

$$
\begin{gathered}
Y_{k}=\left[y_{k}-\bar{v}, y_{k}-\underline{v}\right]=\left[x_{k}^{*}+\bar{v}-\bar{v}, x_{k}^{*}+\bar{v}-\underline{v}\right]=\left[x_{k}^{*}, x_{k}^{*}+\bar{v}-\underline{v}\right], \\
Y_{k+1}=\left[y_{k+1}-\bar{v}, y_{k+1}-\underline{v}\right]=\left[x_{k+1}^{*}+\underline{v}-\bar{v}, x_{k+1}^{*}+\underline{v}-\underline{v}\right]=\left[x_{k+1}^{*}+\underline{v}-\bar{v}, x_{k+1}^{*}\right] .
\end{gathered}
$$

Consider the computation of the interval estimate $X_{k}(6)$ :

$$
X_{k}=X_{k / k-1} \cap Y_{k}=\left[\underline{x}_{k / k-1}, \bar{x}_{k / k-1}\right] \cap\left[x_{k}^{*}, x_{k}^{*}+\bar{v}-\underline{v}\right]=\left[x_{k}^{*}, a\right],
$$

where $a=\min \left\{\bar{x}_{k / k-1}, x_{k}^{*}+\bar{v}-\underline{v}\right\}$. Thus, at time step $k$ the true value $x_{k}^{*}$ of the state $x_{k}$ is the lower bound of the interval estimate $X_{k}: \underline{x}_{k}=x_{k}^{*}$.

Since $f$ is monotone increasing in interval $X_{k / k-1}$, it follows that $f$ is also monotone increasing in interval $X_{k} \subseteq X_{k / k-1}$. Therefore, at time step $k+1$ the true value $x_{k+1}^{*}$ of the state $x_{k+1}$ is the lower bound of the predicted state set $X_{k+1 / k}$ :

$$
X_{k+1 / k}=\left[f\left(x_{k}^{*}, \lambda\right), f(a, \lambda)\right]=\left[x_{k+1}^{*}, f(a, \lambda)\right] .
$$


On the other hand, the true value $x_{k+1}^{*}$ is the upper bound of the consistent state set $Y_{k+1}$. Therefore, the interval estimate $X_{k+1}$ consists of a single point which is the true value $x_{k+1}^{*}$ of the state $x_{k+1}$ :

$$
X_{k+1}=X_{k+1 / k} \cap Y_{k+1}=\left\{x_{k+1}^{*}\right\} .
$$

\section{Conclusion}

This paper has studied the accuracy and convergence properties of the MF for chaotic signals. Numerical simulations have confirmed that the proposed algorithm provides more accurate point estimates than usual approach (choice of the middle point of the interval). The MF has also shown a better performance in comparison with the UKF. In addition, one of the key features of the MF is that the prior information about the measurement errors is presented only as intervals of possible values. It is not necessary to know the model of errors or its probabilistic distribution. Thus, the MF is a very promising technique when the measurement errors are biased and non-Gaussian.

The work was supported by Act 211 Government of the Russian Federation, contract № 02.A03.21.0011.

\section{References}

1. Stepanov O.A. Kalman Filtering: Past and Present. An Outlook from Russia. Gyroscopy and Navigation, 2011, vol. 2, issue 2, pp. 99-110. doi: 10.1134/S2075108711020076.

2. Lefebvre T., Bruyninckx H., De Schutter J. Kalman Filters for Non-Linear Systems: A Comparison of Performance. International Journal of Control, 2004, vol. 77, no. 7, pp. 639-653. doi: 10.1080/00207170410001704998.

3. Simon D. Optimal State Estimation: Kalman, H-infinity, and Nonlinear Approaches. John Wiley \& Sons, 2006. doi: 10.1002/0470045345.

4. Combettes P.L. The Foundations of Set Theoretic Estimation. Proceedings of the IEEE, 1993, vol. 81, no. 2, pp. 182-208. doi: 10.1109/5.214546.

5. Blanchini F., Miani S. Set-Theoretic Methods in Control. Birkhauser, 2015. doi: 10.1007/978-3-319-17933-9.

6. Kurzhanski A.B., Sugimoto K., Valyi I. Guaranteed State Estimation for Dynamical Systems: Ellipsoidal Techniques. International Journal of Adaptive Control and Signal Processing, 1994, vol. 8, no. 1, pp. 85-101. doi: 10.1002/acs.4480080108.

7. Chisci L., Garulli A., Zappa G. Recursive State Bounding by Parallelotopes. Automatica, 1996, vol. 32, no. 7, pp. 1049-1055. doi: 10.1016/0005-1098(96)00048-9.

8. Kieffer M., Jaulin L., Walter E. Guaranteed Recursive Nonlinear State Estimation Using Interval Analysis. Proceedings of the IEEE Conference on Decision and Control, 1998, vol. 4, pp. 3966-3971. doi: 10.1109/CDC.1998.761917. 
9. Alamo T., Bravo J.M., Camacho E.F. Guaranteed State Estimation by Zonotopes. Automatica, 2005, vol. 41, no. 6, pp. 1035-1043. doi: 10.1016/j.automatica.2004.12.008.

10. Leung H., Zhu Z., Ding Z. An Aperiodic Phenomenon of the Extended Kalman Filter in Filtering Noisy Chaotic Signals. IEEE Transactions on Signal Processing, 2000, vol. 48, no. 6, pp. 1807-1810. doi: 10.1109/78.845941.

11. Feng J., Fan H., Tse C.K. Convergence Analysis of the Unscented Kalman Filter for Filtering Noisy Chaotic Signals. Proceedings of the IEEE International Symposium on Circuits and Systems, 2007, pp. 1681-1684. doi: 10.1109/ISCAS.2007.377916.

12. Wang S., Feng J., Tse C.K. Analysis of the Characteristic of the Kalman Gain for 1-D Chaotic Maps in Cubature Kalman Filter. IEEE Signal Processing Letters, 2013, vol. 20, no. 3, pp. 229-232. doi: 10.1109/LSP.2013.2241424.

13. Silva C.P., Young A.M. Introduction to Chaos-Based Communications and Signal Processing. Proceedings of the IEEE Aerospace Conference, 2000, vol. 1, pp. 279-299. doi: 10.1109/AERO.2000.879402.

14. Andreyev Y.V., Dmitriev A.S., Efremova E.V., Anagnostopoulos A.N. Chaotic Signal Processing: Information Aspects. Chaos, Solitons \& Fractals, 2003, vol. 17, no. 2-3, pp. 531-544. doi: 10.1016/S0960-0779(02)00396-X.

15. Feng J.C., Tse C.K. Reconstruction of Chaotic Signals with Applications to ChaosBased Communications. World Scientific, 2008. doi: 10.1142/6585.

16. Nakamura T., Hirata Y., Judd K., Kilminster D., Small M. Improved Parameter Estimation from Noisy Time Series for Nonlinear Dynamical Systems. International Journal of Bifurcation and Chaos, 2007, vol. 17, no. 5, pp. 1741-1752. doi: 10.1142/S021812740701804X.

17. Devaney R.L. An Introduction to Chaotic Dynamical Systems. Addison-Wesley, 1989.

18. Sheludko A.S., Shiryaev V.I. Minimax Filtering Algorithm for One-Dimensional Chaotic Process. Mekhatronika, Avtomatizatsiya, Upravlenie, 2014, no. 5, pp. 8-12. (in Russian)

Anton S. Sheludko, Assistant, Department of Applied Mathematics and Programming, South Ural State University (Chelyabinsk, Russian Federation), sheludkoas@susu.ru.

Received June 1, 2018. 


\title{
О ТОЧНОСТИ И СХОДИМОСТИ АЛГОРИТМА МИНИМАКСНОЙ ФИЛЬТРАЦИИ ДЛЯ ХАОТИЧЕСКОГО СИГНАЛА
}

\section{A. С. Шелудъко}

\begin{abstract}
Рассматривается задача фильтрации для дискретного сигнала, который является реализацией одномерного хаотического отображения. Задача фильтрации состоит в нахождении оценок переменной состояния хаотического отображения по зашумленным измерениям. В рамках гарантированного подхода к решению задачи оценивания разработан алгоритм минимаксной фильтрации. Гарантированный подход основан на множественном представлении неопределенности о неизвестных переменных в модели. Предполагается, что априорная информация о начальном состоянии и ошибках измерений представлена в виде множеств (интервалов) возможных значений. Предложенный алгоритм основан на интервальном анализе и представляет собой рекуррентную процедуру вычисления множественных и точечных оценок переменной состояния хаотического отображения. Построение множественной оценки состоит из трех шагов (прогноз, измерение и коррекция), аналогичных этапам построения информационного множества для линейной динамической системы. Для нахождения точечных оценок используется алгоритм, основанный на аналогии с уравнениями фильтра Калмана. В данной работе исследуется точность и сходимость алгоритма минимаксной фильтрации. Основные цели исследований: подтвердить эффективность предложенного алгоритма нахождения точечных оценок, сравнить точность результатов алгоритма минимаксной фильтрации и алгоритма UKF (модификации фильтра Калмана для нелинейных моделей), представить достаточные условия нахождения точных оценок переменной состояния. Вычислительная схема алгоритма минимаксной фильтрации и численные эксперименты приводятся для квадратичного отображения.
\end{abstract}

Ключевые слова: хаотический сигнал; задача фильтрачии; гарантированное оценивание; интервальная оценка.

\section{Литература}

1. Stepanov, O.A. Kalman Filtering: Past and Present. An Outlook from Russia O.A. Stepanov // Gyroscopy and Navigation. - 2011. - V. 2, issue 2. - P. 99-110.

2. Lefebvre, T. Kalman Filters for Non-Linear Systems: A Comparison of Performance / T. Lefebvre, H. Bruyninckx, J. De Schutter // International Journal of Control. 2004. - V. 77, № 7. - P. 639-653.

3. Simon, D. Optimal State Estimation: Kalman, H-infinity, and Nonlinear Approaches / D. Simon. - John Wiley \& Sons, 2006.

4. Combettes, P.L. The Foundations of Set Theoretic Estimation / P.L. Combettes // Proceedings of the IEEE. - 1993. - V. 81, № 2. - P. 182-208.

5. Blanchini, F. Set-Theoretic Methods in Control / F. Blanchini, S. Miani. - Birkhauser, 2015.

6. Kurzhanski, A.B. Guaranteed State Estimation for Dynamical Systems: Ellipsoidal Techniques / A.B. Kurzhanski, K. Sugimoto, I. Valyi // International Journal of Adaptive Control and Signal Processing. - 1994. - V. 8, № 1. - P. 85-101. 
7. Chisci, L. Recursive State Bounding by Parallelotopes / L. Chisci, A. Garulli, G. Zappa // Automatica. - 1996. - V. 32, № 7. - P. 1049-1055.

8. Kieffer, M. Guaranteed Recursive Nonlinear State Estimation Using Interval Analysis / M. Kieffer, L. Jaulin, E. Walter // Proceedings of the IEEE Conference on Decision and Control. - 1998. - V. 4. - P. 3966-3971.

9. Alamo, T. Guaranteed State Estimation by Zonotopes / T. Alamo, J.M. Bravo, E.F. Camacho // Automatica. - 2005. - V. 41, № 6. - P. 1035-1043.

10. Leung, H. An Aperiodic Phenomenon of the Extended Kalman Filter in Filtering Noisy Chaotic Signals / H. Leung, Z. Zhu, Z. Ding // IEEE Transactions on Signal Processing. - 2000. - V. 48, № 6. - P. 1807-1810.

11. Feng, J. Convergence Analysis of the Unscented Kalman Filter for Filtering Noisy Chaotic Signals / J. Feng, H. Fan, C.K. Tse // Proceedings of the IEEE International Symposium on Circuits and Systems. - 2007. - P. 1681-1684.

12. Wang, S. Analysis of the Characteristic of the Kalman Gain for 1-D Chaotic Maps in Cubature Kalman Filter / S. Wang, J. Feng, C.K. Tse // IEEE Signal Processing Letters. - 2013. - V. 20, № 3. - P. 229-232.

13. Silva, C.P. Introduction to Chaos-Based Communications and Signal Processing C.P. Silva, A.M. Young // Proceedings of the IEEE Aerospace Conference. - 2000. V. 1. - P. 279-299.

14. Andreyev, Y.V. Chaotic Signal Processing: Information Aspects / Y.V. Andreyev, A.S. Dmitriev, E.V. Efremova, A.N. Anagnostopoulos // Chaos, Solitons \& Fractals. 2003. - V. 17, № 2-3. - P. 531-544.

15. Feng, J.C. Reconstruction of Chaotic Signals with Applications to Chaos-Based Communications / J.C. Feng, C.K. Tse. - World Scientific, 2008.

16. Nakamura, T. Improved Parameter Estimation from Noisy Time Series for Nonlinear Dynamical Systems / T. Nakamura, Y. Hirata, K. Judd, D. Kilminster, M. Small // International Journal of Bifurcation and Chaos. - 2007. - V. 17, № 5. - P. 1741-1752.

17. Devaney, R.L. An Introduction to Chaotic Dynamical Systems / R.L. Devaney. Addison-Wesley, 1989.

18. Шелудько, А.С. Алгоритм минимаксной фильтрации для одномерного хаотического процесса / А.С. Шелудько, В.И. Ширяев // Мехатроника, автоматизация, управление. - 2014. - № 5. - С. 8-12.

Шелудъко Антон Сергеевич, ассистент, кафедра прикладной математики и программирования, Южно-Уральский государственный университет (г. Челябинск, Российская Федерачия), sheludkoas@susu.ru.

Поступила в редакцию 1 июня 2018 г. 